\title{
Pericardiodiaphragmatic rupture and cardiac herniation after multiple blunt trauma: Diagnostic and therapeutic difficulties
}

\author{
Z. Witkowski, PhD, ${ }^{\text {a }}$ J. Lasek, MD, ${ }^{a}$ M. Wujtewicz, PhD, ${ }^{\text {b } M . ~ S t a s i a k, ~ P h D, ~}{ }^{a}$ W. Marks, MD, ${ }^{a}$ and
}

A. Kawecka, MD, ${ }^{a}$ Gdańsk, Poland

$\mathrm{P}$

ericardial rupture with cardiac herniation $(\mathrm{CH})$ after severe multiple trauma is observed rarely and frequently is diagnosed during postmortem examinations. Fulda and associates ${ }^{1}$ described 4 patients with pericardiodiaphragmatic rupture (PDR); however, no $\mathrm{CH}$ was observed among 20000 trauma patients in their materials. The overall morbidity rate in patients with PDR is high, mainly in multiply injured victims. ${ }^{1,2}$ We describe a report of a successful treatment of a multiply injured patient who sustained PDR and $\mathrm{CH}$.

\section{Clinical Summary}

A 55-year-old woman was transferred to our university trauma surgery unit from a local hospital about 14 days after a car accident in which 3 other persons died. After the crash, the patient was in shock (blood pressure, $60 / 0 \mathrm{~mm} \mathrm{Hg}$; heart rate, $>140$ beats $/ \mathrm{min}$ ). The initial chest radiograph showed pneumothorax and rib fractures VI-X of the left side and fractures of both clavicles. Left-side pleural drainage was installed, and an emergency laparotomy was introduced. It revealed a diaphragmatic rupture about $15 \mathrm{~cm}$ long on the left side, with herniation of a severely injured spleen and large intestine (flexura lienalis) into the pleural cavity. Because of the severe damage, splenectomy was performed, and the large intestine was brought back to the abdominal cavity. The left-side diaphragmatic defect was repaired with interrupted sutures. In addition, the patient sustained brain contusion, fracture of the radial bone, and fractures of both clavicles. Initially the Injury Severity Score was estimated at 34 points. In the postoperative course, tachycardia (heart rate up to 200 beats/min) and high blood pressure (190/100 $\mathrm{mm} \mathrm{Hg}$ ) and ventricular extrasystoles (bigeminy) were observed. The echocardiographic examination showed enlargement of the right cardiac atrium and chamber. After 14 days, the patient was transferred to our department. At admission, the patient presented with cardiac ventricular extrasystolic rhythm. A new echocardiogram revealed a layer of pericardial fluid (1.1$2.4 \mathrm{~cm}$ wide). Echocardiography that was performed 3 days later showed pericardial fluid behind the left ventricular chamber ( 3.5 $\mathrm{cm}$ wide). Computed tomography (CT) of the chest revealed signs

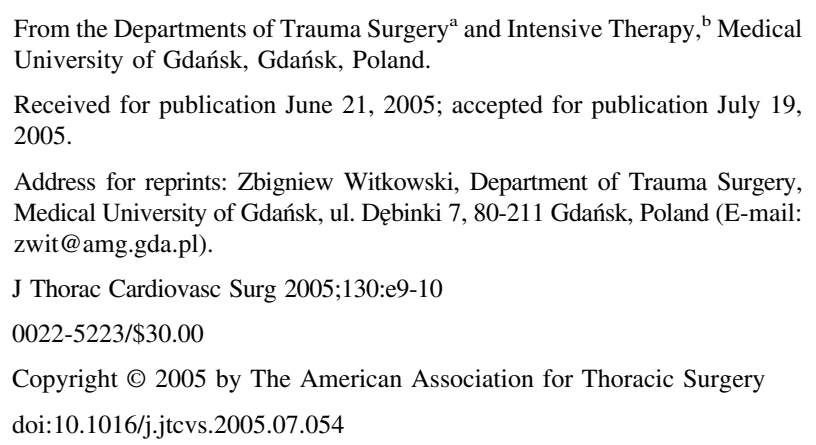

of an atelectasis of the left lower lung lobe and blood in the left pleural cavity and hemopericardium with the presence of a fluid layer to $0.9 \mathrm{~cm}$ wide. The heart showed some rotation and displacement to the left (Figure 1). It was decided to operate on the patient again 19 days after the initial trauma and operation. A left posterolateral thoracotomy was performed. It was found that the heart was completely herniated through the ruptured pericardium into the left pleural space. The defect in the pericardium extended from the superior vena cava down to the diaphragm (Figure 2). The heart appeared dilated and contused. The pericardial defect was closed with nonresorbable Marlex mesh graft. In addition, intramedullar osteosynthesis of the fractured clavicles was performed with Kirschner wires. The postoperative course was not complicated. Single extrasystoles were noted some days after the operation. Echocardiography was performed on the sixth postoperative day and 8 weeks later showed minimal enlargement of the right chambers. The patient returned to normal activities after 2 months and remains under the care of our outpatient clinic, with no serious complaints for 8 months since the multiple trauma.

\section{Discussion}

PDR is a rare ${ }^{1-3}$ clinical finding, but it should be suspected in patients after severe blunt trauma to the chest. It is very difficult to diagnose on clinical grounds. Historically, in 1864, MorelLavallee reported a murmur sounding like a splashing mill wheel ("bruit de Moulin") in a patient with a pericardial rupture. ${ }^{4}$ The lacerations of pericardium might be situated in 3 regions $^{3,4}$ : the diaphragmatic, superior mediastinal, and left or right pleuropericardial regions. If the diaphragmatic pericardium and diaphragm are ruptured simultaneously, the abdominal contents might herniate into the pericardial sac and cause cardiac compression with

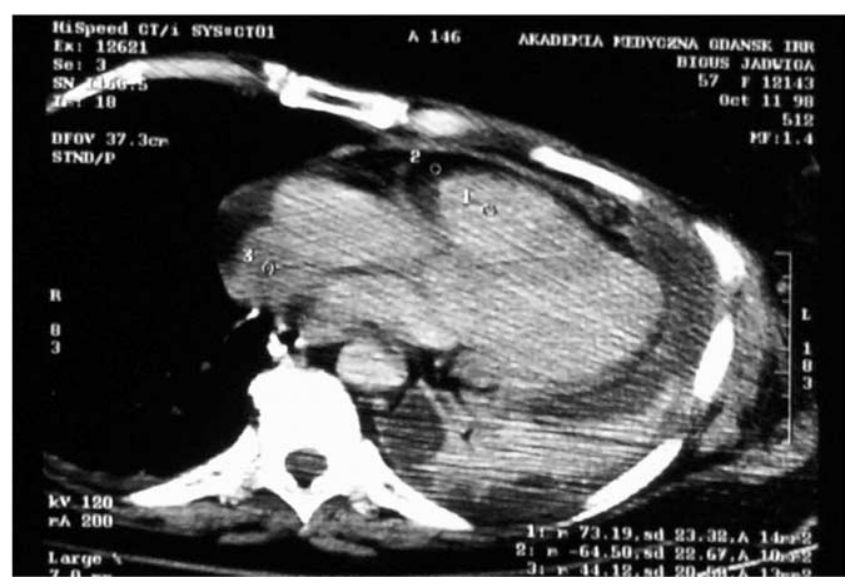

Figure 1. CT scan of the chest in a patient with PDR. 


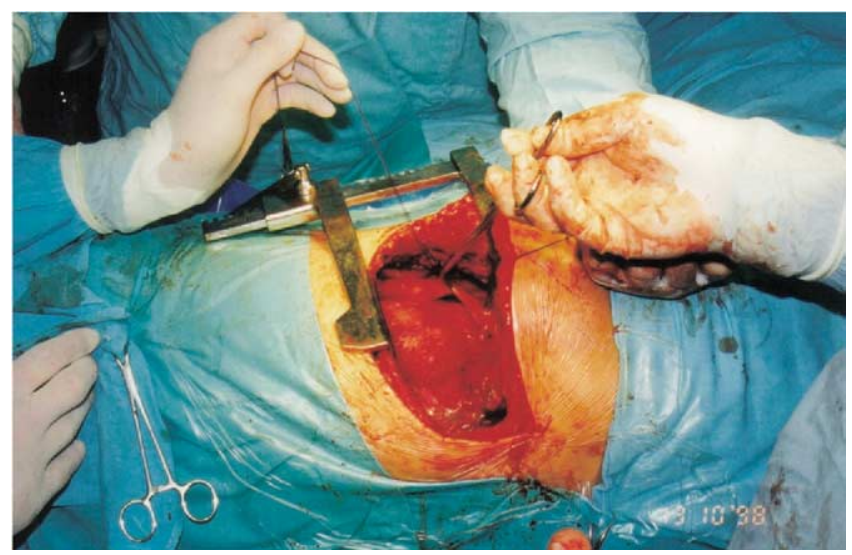

Figure 2. Ruptured pericardium with the herniated heart found during thoracotomy.

cardiogenic shock. If the pleural pericardium and diaphragm are injured, the heart might herniate into the pleural space, resulting in heart constriction, fatal strangulation, or even total torsion of the great vessels, resulting in sudden death. The diagnosis ${ }^{1,4,5}$ is accomplished by means of chest radiography or CT when there is displacement of the heart. ${ }^{5}$ Today, in stable patients video-assisted thoracoscopy (VATS) can be used. Electrocardiographic signs are nonspecific for $\mathrm{CH}$. The results of initial radiographic examinations seem to be normal and very rarely might show a pneumomediastinum associated with hemopneumothorax and hemopericardium. Transesophageal echocardiography might reveal a pericardial lesion. CT appears decisive in the diagnosis because it noninvasively visualizes the pericardium and reveals pericardial rupture..$^{5}$ When the diaphragmatic tear is large, the intestinal obstruction might never appear. The patient might demonstrate nonspecific abdominal and chest symptoms. As a rule, all patients after severe blunt trauma should undergo $\mathrm{CT}^{5}$ of the chest and echocardiography if plain chest radiographic examinations show an enlargement of the mediastinum.

Our report confirms considerable diagnostic difficulties in the case of PDR with $\mathrm{CH}$. Some of these difficulties are connected with the fact that immediately after trauma the treatment was started in a small local hospital without modern diagnostic facilities. It seems probable that the pericardial rupture was small or could be misdiagnosed by the surgeon operating within the abdominal cavity through the ruptured left diaphragmatic dome. Probably later, as the pericardial rupture grew larger, the heart herniated into the left pleural cavity and caused several clinical symptoms. However, with proper monitoring, even in the case of delayed diagnosis of PDR with $\mathrm{CH}$, the patient can be successfully treated with surgical repair of the pericardial sac.

\section{References}

1. Fulda G, Brathwaite CE, Rodriguez A, et al. Blunt traumatic rupture of the heart and pericardium: a ten-year experience (1979-1989). J Trauma. 1989;31:167-73.

2. Sharma P. Pericardio-diaphragmatic rupture: five new cases and literature review. J Emerg Med. 1999;17:963-8.

3. Van Tornout F, Van Leuven M, Parry W. Pericardio-diaphragmatic avulsion and concomitant ruptured central tendon of the diaphragm. Eur J Cardiothorac Surg. 2004;26:655-7.

4. Beless DJ, Organ BC. Delayed presentation of intrapericardial diaphragmatic hernia: unusual cause of colon obstruction. Ann Emerg Med. 1991;20:415-7.

5. Rashid MA, Lund JT. Blunt traumatic pericardial rupture: a diagnostic challenge. Eur J Trauma. 2003;6:403-5. 